\title{
Unusual Presentation of Osteochondroma as Bunionette: Case Report with Review of Literature
}

\author{
Mohammad Eid ${ }^{*}$, Mohammad Alsafi², Sager Hanna ${ }^{3}$ and Aliaa \\ Khaja ${ }^{3}$ \\ ${ }^{1}$ Foot/Ankle Specialist, Al Razi Orthopedic Hospital, Sabah Medical Area, Kuwait \\ City, Kuwait \\ ${ }^{2}$ Orthopedic Surgeon (Senior), Al Razi Orthopedic Hospital, Sabah Medical Area, \\ Kuwait City, Kuwait \\ ${ }^{3}$ Orthopedic Surgeon (Resident), Al Razi Orthopedic Hospital, Sabah Medical \\ Area, Kuwait City, Kuwait \\ *Corresponding Author: Mohammad Eid, Foot/Ankle Specialist, Al Razi \\ Orthopedic Hospital, Sabah Medical Area, Kuwait City, Kuwait.
}

Received: March 16, 2020

Published: March 31, 2020

(C) All rights are reserved by Mohammad

Eid., et al.

\begin{abstract}
Introduction: Osteochondromas are bone lesions presenting in benign form. The lesions originate from aberrant cartilage. Bunionette on the other hand, presents in a lateral prominence of the fifth metatarsal head, inducing a callus. The varus, infraductus, or supraductus form is determined by toe malpositioning. Osteochondromas are one of the commonest bone lesions, but their occurrence in the ankle and foot is quite rare.

Case Presentation: This article reports the case of a twelve-year-old boy of Arabic ethnic background, who presented with lateral foot pain diagnosed as a bunionette. The patient underwent surgical excision with the bone presenting a suspicious consistency. The excised tissue was sent for histopathology and it came back as an osteochondroma.

Conclusion: To the best of our knowledge, there are no reports of osteochondromas presenting as a bunionette. It is an extremely rare condition. We, therefore, present this case as an instance of chronic complication of osteochondral lesions. This rare case can help shed light on such peculiar presentations to Orthopedic Surgeons, Foot/Ankle Specialists and Orthopedic Oncologists who would have such cases referred to them.
\end{abstract}

Keywords: Presentation; Osteochondroma; Bunionette

\section{Introduction}

Bunionette is a deformity that presents in a lateral prominence of the fifth metatarsal head, resulting in a callus on the plantar side or the lateral side. It has a higher frequency in female subjects than in male subjects; research has shown that it is three to six times higher in females than in males [1]. There is the absence of exostosis of the metatarsal head, just as in hallux valgus, but instead lateral migration, at times linked with a morphologic abnormality. M.J. Coughlin clearly distinguished the three types of bunionettes based on the work of H.L. Du Vries, with type 1 associated with a prominent lateral condyle and an increased fifth metatarsal head volume (16 - 33\%); type 2 characterized by bow deformity causing the lateral condyle to become significantly prominent (10\%); while type 3 is characterized by a high fourth metatarsal-fifth metatarsal angle without any distal fifth metatarsal deformity (57 - 74\%) [2,3]. There may be congenital varus overlap of the fifth toe, in supraductus or infraductus.

Osteochondromas are very common bone tumors with a 30 $40 \%$ incidence rate [4]. These tumors are benign and are believed to be caused by a perichondral congenital defect [4]. The lesions occur close to the growth plates of long bones and in most cases, form around the knee or shoulder [4]. Very little research has been done concerning the involvement of other anatomic sites. These may originate in bones that are formed from cartilage [5]. Three to four percent of all osteochondromas are of the scapular type [6]. The occurrence of osteochondromas around the small bones of the foot is also rare.

\section{Case Presentation}

A twelve-year old male presented with a bunionette on the left foot. The patient also experienced discomfort with weight-bearing on the same foot. Pain and stiffness worsened progressively over four to five months. Relief from disability and pain was provided with Non-steroidal anti-inflammatory drugs (NSAID) and bracewear (non-operative modalities). The patient could not partake in high-impact activities nor activities on uneven ground due to the pain.

\section{Investigation and treatment}

Physical examination revealed a near-total restriction of subtalar motion which was accompanied with extreme pain on passive and active foot eversion and inversion. In addition, we observed a bony swelling over the lateral forefoot. Radiographs showed a bony lesion extending from the neck of the fifth metatarsal which we 
thought was an osteochondroma (Figure 1). Further workup included a Magnetic Resonance Imaging (MRI) of the Left foot which showed a bony exostosis with a $2 \mathrm{~mm}$ cartilage cap suggesting an osteochondroma (Figure 2). Surgical excision of the exostosis and subsequent histopathological examination confirmed this diagnosis.

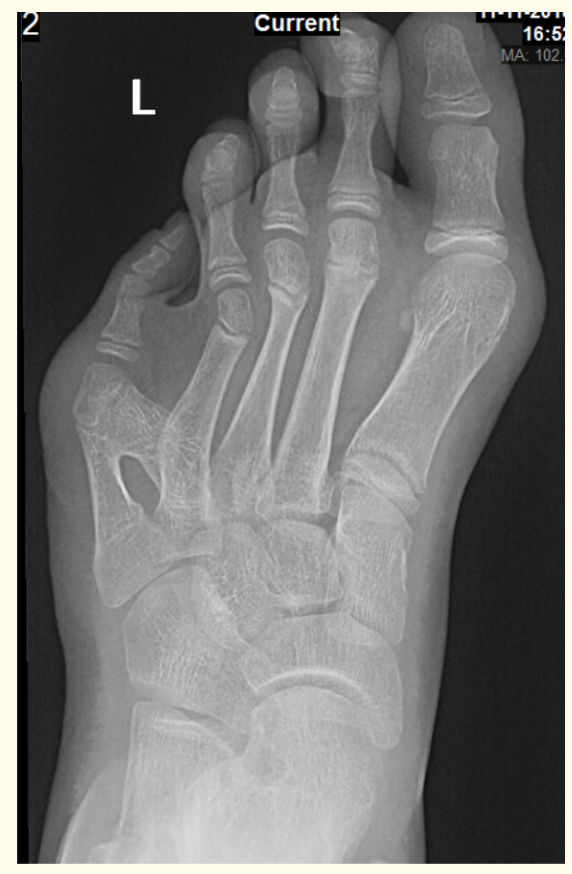

Figure 1: Standing anterior-posterior left foot radiograph. This is the radiograph taken on patient initial presentation when he complained of lateral-sided foot pain and swelling.

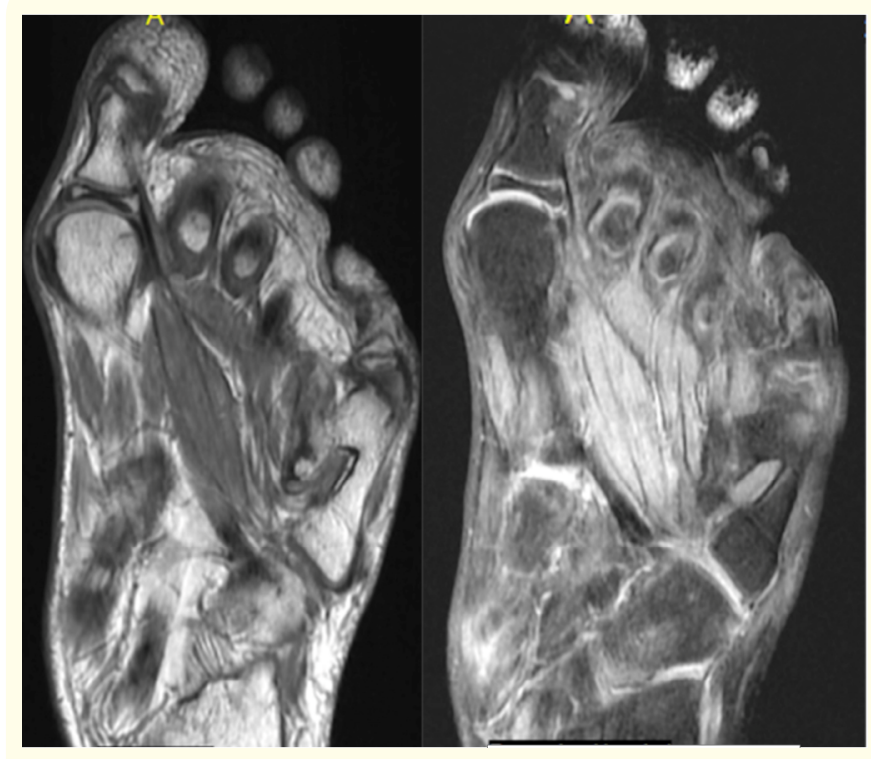

Figure 2: Magnetic resonance image (MRI) T1 and T2 Axial cut of the left foot. This is the MRI image showing the bony exostosis at the fifth metatarsal, Left foot. It was done to

help determine the nature of our findings in our first radiograph. This is pre-operative as part of the assessment.

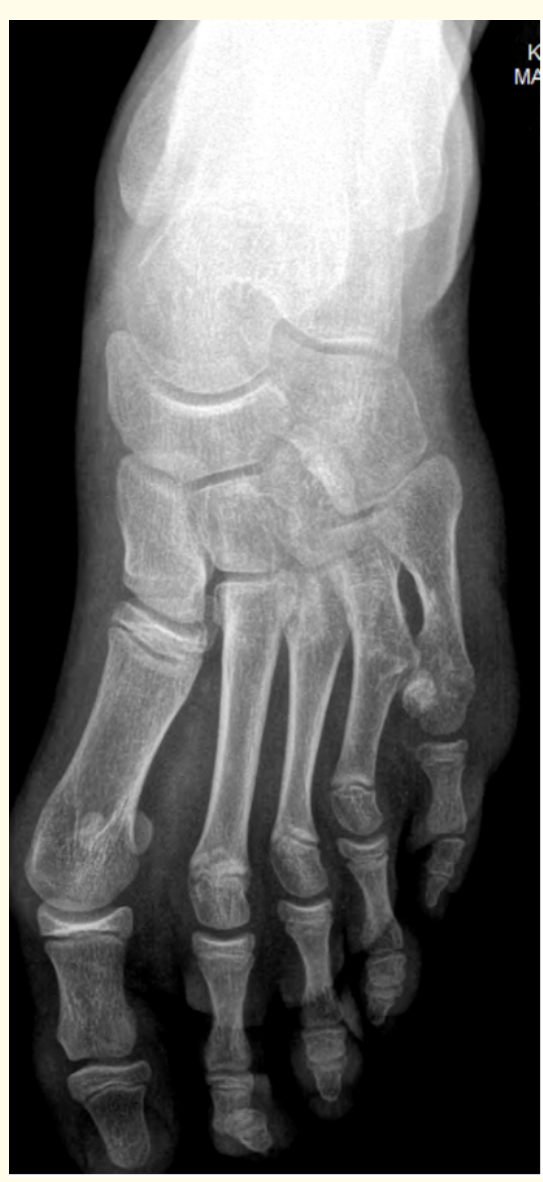

Figure 3: Standing anterior-posterior left foot radiograph at one-year post-operative follow up. This radiograph shows the foot one year post-operatively with correction of the bunionette

deformity and patient has resolution of his symptoms.

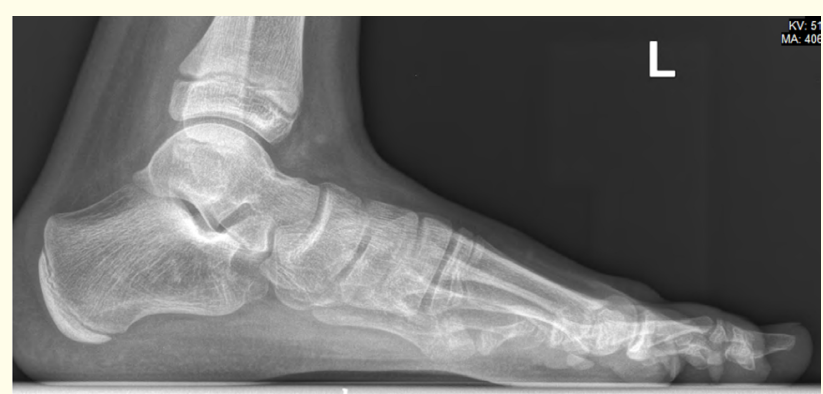

Figure 4: Standing lateral left foot radiograph at one-year post-operative follow up. This radiograph shows the foot from a lateral view as the patient's symptoms have been resolved, and no deformity nor exostosis is seen.

\section{Discussion}

Osteochondromas are a very common bone tumor of benign setting. They make up $30-40 \%$ of cases of benign bone lesions. Research has also shown that they make up $15 \%$ of all bone-related tumors. Osteochondromas represent dislocation of the cartilage of the growth plate where there is normal longitudinal growth adjacent to the lesion's centripetal growth in the bone's metaphyseal 
region. After the closure of the growth plate, growth of the lesion ceases and the osteochondroma cartilage cap matures to a thickness of $2 \mathrm{~mm}$ [7]. If there is a growth of lesion in adulthood, they are usually a representation of the malignant transformation of the cartilage into chondrosarcoma [8-10]. In many cases, osteochondroma lesions grow from locations that may be termed 'metaphyseal', away from the adjacent joint. It should be noted that Fairbank's disease or Dysplasia Epiphysealis Hemimelica are variants of osteochondroma with intra-articular lesions. The lesions, in this case, grow adjacent to the joint cartilage [11].

Several case reports have demonstrated the concurrent development of osteochondroma of adjacent metaphyseal regions, resulting in "kissing" lesions whose growth occurs adjacent to an affected joint, resulting in presentation and pain in childhood [9]. There have been literature reports of osteochondromas occurring adjacent to a periosteal chondroma thus giving rise to a kissing lesion [10].

In most cases, these lesions present with pain or innocuous swelling, and in some instances with mechanical compression or restriction of movement. Also, they may cause joint deformity, intra-articular loose body, inequality of limb length, or peroneal spastic flatfoot [12].

In children, the condition should be managed conservatively, as surgical intervention for intra-articular lesions may cause secondary arthrosis. For juxta-articular or metaphyseal lesions, early surgical intervention is recommended to avoid complications associated with deformity and growth. In adults presenting with a single osteochondroma, surgery is recommended due to the risk of malignant growth or transformation beneath a large tendinous sleeve at the metaphyseal insertion when a snapping syndrome may develop [13].

Cases of osteochondroma around foot metatarsals are very rare. Very few case reports about this condition have been published to date. Publications on osteochondroma presenting as a bunionette are non-existent to the best of our knowledge. A 2010 report by Yildirim., et al. [14], gave an instance of a lone giant osteochondroma in an army recruit. The osteochondroma occurred in the fifth metatarsal. A 2015 report by Shtofmakher., et al. [15] gave instances of symptomatic osteochondroma originating from the first metatarsal. Weight-bearing caused pain in these patients. The same experience was encountered in our case. Weight-bearing caused an aching pain on the plantar aspect of the left foot of our patient.

\section{Conclusion}

The symptoms of osteochondroma vary, although this depends on the site of occurrence. Differential diagnosis of osteochondromas should be considered for patients who present with bony swellings around small, flat bones of the feet. Considering such differentials will enable treating surgeons to correctly choose the treatment(including surgery) for such cases and individualize the management according to the specific patient's needs and expectations.

\section{Consent}

The authors declare that informed consent has been granted for this case.

\section{Competing Interests}

The authors declare that they have no competing interests.

\section{Authors' Contributions}

M.E.: The treating surgeon, he was in charge of nonoperative as well as operative management and the patient follows up in his clinic. He presented this case as a case of interest for the report.

M.A.: He was also actively involved in treating the patient and following him up. He also had the task of doing a literature review of similar cases that have relevance to our report.

S.H.: Revised the final draft of the manuscript and collected the images used in the report. He also helped in going over the references used in this report.

A.K.: Study design as well as writing the first draft and reviewing the manuscript. She was also responsible for keeping the team on schedule with respect to timeframe available and allocation of roles.

\section{Bibliography}

1. TS Roukis. "The tailor's bunionette deformity: a field guide to surgical correction". Clinics in Podiatric Medicine and Surgery 22 (2005): 223-245.

2. MJ Coughlin. "Treatment of bunionette deformity with longitudinal diaphyseal osteotomy with distal soft tissue repair". Foot Ankle 11 (1991): 195-203.

3. HL Du Vries. "Surgery of the foot". (2 $2^{\text {nd }}$ edition), CV Mosby, St Louis (1965): 456-462.

4. Puri A and Agrawal MG. "Current concepts in bone and soft tissue tumours". Textbook of orthopaedic oncology. Paras Medical Books Pvt. Ltd: 72-77.

5. Kumar Y., et al. "Rahul Sessile Osteochondroma of Scapula, a Rare Case Report". Journal of Clinical and Diagnostic Research 8.3 (2014): 174-175.

6. Mahajan S., et al. "Scapula a rare localization of osteochondroma: a case report". The Internet Journal of Orthopedic Surgery 14.1 (2008).

7. Marco RA., et al. "Cartilage Tumors: Evaluation and Treatment". The Journal of the American Academy of Orthopaedic Surgeons 8 (2000): 292-304.

8. Ahmed AR., et al. "Secondary chondrosarcoma in osteochondroma: report of 107 patients". Clinical Orthopaedics 411 (2003): 193-206. 
9. Murphey MD., et al. "Imaging of osteochondroma: variants and complications with radiologic-pathologic correlation". Radiographics 20 (2000): 1407-1434.

10. Wuisman PIJ., et al. "Secondary chondrosarcoma in osteochondromas: Medullary extension in 15 of 45 cases". Acta Orthopaedica Scandinavica 68 (1997): 396-400.

11. Staals EL., et al. "Dedifferentiated chondrosarcomas arising in preexisting osteochondromas". Journal of Bone and Joint Surgery America 89.5 (2007): 987-993.

12. Blair J., et al. "Peroneal spastic flatfoot caused by a talar osteochondral lesion: a case report". Foot and Ankle International 28.6 (2007): 724-726.

13. Christopher G MD., et al. "Osteochondromas of the subtalar joint: A case study". The Foot and Ankle Online Journal 10.3 (2017): 2.

14. Yildırım C., et al. "Giant solitary osteochondroma arising from the fifth metatarsal bone: A Case Report". The Journal of Foot and Ankle Surgery 49 (2010): 298.

15. Shtofmakher G., et al. "Multiple osteocartilaginous exostoses of the lower extremity: A case report". The Foot 25.1 (2015): 62-65.

\section{Assets from publication with us}

- Prompt Acknowledgement after receiving the article

- Thorough Double blinded peer review

- Rapid Publication

- Issue of Publication Certificate

- High visibility of your Published work

Website: https://www.actascientific.com/

Submit Article: https://www.actascientific.com/submission.php

Email us: editor@actascientific.com

Contact us: +919182824667 